\title{
QUASIDIAGONALITY OF DIRECT SUMS OF WEIGHTED SHIFTS
}

\author{
SIVARAM K. NARAYAN
}

\begin{abstract}
Let $\mathscr{H}$ be a separable Hilbert space. A bounded linear operator $A$ defined on $\mathscr{H}$ is said to be quasidiagonal if there exists a sequence $\left\{P_{n}\right\}$ of projections of finite rank such that $P_{n} \rightarrow I$ strongly and $\left\|A P_{n}-P_{n} A\right\| \rightarrow 0$ as $n \rightarrow \infty$.

We give a necessary and sufficient condition for a finite direct sum of weighted shifts to be quasidiagonal. The condition is stated using a marked graph (a graph with a $(0),(+)$ or $(-)$ attached to its vertices) that can be associated with the direct sum.
\end{abstract}

\section{INTRODUCTION}

In 1973, R. A. Smucker gave a characterization of quasidiagonality for weighted shifts in terms of their weight sequences $[16,17]$. Smucker also showed that quasidiagonality is not invariant under similarity transformations.

There are many examples of operators $A$ and $B$, neither of which is quasidiagonal, such that their direct sum $A \oplus B$ is quasidiagonal. One such example is the direct sum of the unilateral shift and its adjoint. In the main theorem we give a necessary and sufficient condition for a finite direct sum of weighted shifts to be quasidiagonal.

$\S 1$ contains some known results about quasidiagonality and Smucker's theorem. The statement of the main theorem is given in $\S 2$. In $\S 3$ we state and prove some results about certain "marked graphs". These results are then used to prove the sufficiency of our condition in $\S 4$. In $\S 5$ the necessity of our condition is proved.

\section{Preliminaries}

Throughout $\mathscr{H}$ will denote a separable Hilbert space. The collection of bounded linear operators on $\mathscr{H}$ will be denoted by $\mathscr{B}(\mathscr{H})$. The term 'projection' will mean an orthogonal projection on $\mathscr{H}$. A direct sum of operators $A$ and $B$ in $\mathscr{B}(\mathscr{H})$ will be denoted by $A \oplus B$. For other terminology and standard results in operator theory we refer to $[7,11]$.

Received by the editors August 19, 1988 and, in revised form, May 17, 1990.

1980 Mathematics Subject Classification (1985 Revision). Primary 47B37; Secondary 46L99, 05 C99.

Key words and phrases. Quasidiagonality, weighted shifts, marked graphs, crossed products of $C^{*}$-algebras.

Much of the following material is part of the author's Ph.D. dissertation written under the guidance of Professor Lawrence G. Brown at Purdue University. 
1.1. Quasidiagonal operators. In 1970 , P. R. Halmos defined and studied quasidiagonal operators. The following definition was given in [10].

Definition. An operator $A$ in $\mathscr{B}(\mathscr{H})$ is said to be quasidiagonal if there exists a sequence $\left\{P_{n}\right\}_{1}^{\infty}$ of projections of finite rank such that $P_{n} \rightarrow I$ strongly and $\left\|A P_{n}-P_{n} A\right\| \rightarrow 0$ as $n \rightarrow \infty$.

Other equivalent definitions for quasidiagonality were given by Halmos in [10] (see also [12]).

The collection of quasidiagonal operators on $\mathscr{H}$ will be denoted by $(Q D)$. The following results can be found in $[1,9,10,13,14$ and 16]. These results will be assumed in our work.

1. $(Q D)$ is invariant under unitary equivalence.

2. $(Q D)$ is invariant under compact perturbations.

3. $(Q D)$ is closed under countable direct sums.

4. If $A$ is in $(Q D)$ then every operator in $C^{*}(A)$, the $C^{*}$-algebra generated by $A$, is in $(Q D)$.

5. If $0 \oplus A$ is in $(Q D)$ then $A$ is in $(Q D)$.

6. If $A$ is in $(Q D)$ and is semi-Fredholm then the index of $A$ is zero.

\subsection{Weighted shifts and Smucker's theorem.}

Definition. Let $\mathscr{H}=l^{2}(\mathbf{Z})$ and $\left\{e_{j}\right\}_{j \in \mathbf{Z}}$ be the standard orthonormal basis. If $\left\{w_{j}\right\}_{j \in \mathbf{Z}}$ is a bounded sequence of numbers then a weighted bilateral shift $S$ is defined by the relation

for each $j$ in $\mathbf{Z}$.

$$
S e_{j}=w_{j} e_{j+1}
$$

If $w_{j}=0$ for all $j<0$ then $S$ is written as $0 \oplus S^{\prime}$ where $S^{\prime}$ is said to be a weighted unilateral shift.

If $w_{j}=1$ for all $j$ in $\mathbf{Z}$ then the (unweighted) bilateral shift is denoted by $W$. If $w_{j}=0$ for $j<0$ and $w_{j}=1$ for $j \geq 0$ then the shift is $0 \oplus U$ where $U$ is the (unweighted) unilateral shift.

Because $S$ is unitarily equivalent to the weighted shift with weight sequence $\left\{\left|w_{j}\right|\right\}_{j \in \mathbf{Z}}$ and quasidiagonality is preserved under unitary equivalence we will assume that $S$ has weight sequence $\left\{w_{j}\right\}_{j \in Z}$ with $w_{j} \geq 0$.

Due to their simple structure weighted shifts have been studied extensively. The shifts are often used to provide examples and counterexamples (see [11 and 15]).

We give below Smucker's theorem.

Definition. A sequence $\left\{w_{j}\right\}_{j \in Z}$ of numbers is said to be block balanced if for each positive number $\varepsilon$ and for each positive integer $n$ there exist integers $p$ and $q$ with $p+n<0<q$ and such that $\left|w_{p+k}-w_{q+k}\right|<\varepsilon$ for each $k$ with $0 \leq k \leq n$.

Theorem [16, 17]. A weighted bilateral shift is quasidiagonal if and only if the weight sequence either has zero as a limit point in both directions or is block balanced.

\section{Statement of the Main Theorem}

Assume that $T_{1}, T_{2}, \ldots, T_{k}$ are weighted bilateral shifts defined on $\mathscr{H}=$ $l^{2}(\mathbf{Z})$. Let $\left\{e_{j}\right\}_{j \in \mathbf{Z}}$ be the standard orthonormal basis for $\mathscr{H}$ and define $\mathscr{H}_{+}=$ 
$\operatorname{span}\left\{e_{j} \mid j \geq 0\right\}$ and $\mathscr{H}_{-}=\operatorname{span}\left\{e_{j} \mid j<0\right\}$. For each weighted bilateral shift $T_{i}$ $(1 \leq i \leq k)$ define $T_{i}^{0}$ to be equal to $T_{i}$ except that $T_{i}^{0} e_{-1}=0$. Clearly $T_{i}^{0}$ is obtained by a compact perturbation of $T_{i}$. Then, define the positive end $T_{i+}$ of $T_{i}$ to be $\left.T_{i}^{0}\right|_{\mathscr{H}_{+}}$, the restriction of $T_{i}^{0}$ to $\mathscr{H}_{+}$, and the negative end $T_{i-}$ of $T_{i}$ to be $\left.T_{i}^{0}\right|_{\mathscr{H}_{-}}$, the restriction of $T_{i}^{0}$ to $\mathscr{H}_{-}$.

Let $\bar{T}$ denote the direct sum $T_{1} \oplus T_{2} \oplus \cdots \oplus T_{k}$. We note that the direct sum of the $2 k$ ends of $T_{1}, T_{2}, \ldots, T_{k}$ is a compact perturbation of $\bar{T}$ and will be denoted by $T$. The necessary and sufficient condition for $T$ to be quasidiagonal is given in the theorem via a marked graph $G$ associated with $T$.

Definition. Suppose $\left\{w_{j}^{a}\right\}$ and $\left\{w_{i}^{b}\right\}$ are the weight sequences for two distinct ends $T_{a}$ and $T_{b}$ of $T$. These two ends of $T$ are said to be block balanced if for every positive integer $n$ there exist integers $r$ and $s$ such that

$$
\max _{0 \leq p \leq n}\left|w_{r+p}^{a}-w_{s+p}^{b}\right|<\frac{1}{n} \text {. }
$$

It is permissible to assume that $r, s \rightarrow \pm \infty$, according as the end is positive or negative, as $n \rightarrow \infty$.

Definition. A graph $G$ is associated with $T$ as follows: The vertex set of $G$ consists of the $2 k$ ends of $T$ and an edge is drawn between two vertices if and only if the corresponding ends of $T$ are block balanced.

Definition. The graph $G$ associated with $T$ has a $(0),(+)$, or $(-)$ attached to each of its vertices as follows: $A(0)$ is attached to a vertex if the weight sequence of the corresponding end has zero as a cluster point. Otherwise, a $(+)$ or $(-)$ is attached according as the end is positive or negative respectively. The graph $G$ is now called a marked graph.

Main Theorem. A finite direct sum $\bar{T}$ of weighted bilateral shifts is quasidiagonal if and only if every component of the marked graph $G$ associated with $T$ has either

(i) an equal number of $(+)$ and (-) vertices, or

(ii) $a(0)$ vertex.

Remark. L. G. Brown has shown in [5] the existence of a nonquasidiagonal operator $A$ such that $A \oplus A$ is quasidiagonal. It is a consequence of our theorem that such an $A$ cannot be a finite direct sum of weighted shifts.

\section{SOME RESUlTS ABOUT MARKED GRAPHS}

The first section contains some definitions and terminology in graph theory. In $\S 3.2$ we define a marked graph and prove some lemmas and a theorem. This second section will provide a proper context for the sufficiency proof of the main theorem in $\S 4$.

3.1. Graph theoretic preliminaries. A graph $G$ is an ordered pair of disjoint sets $(V, E)$ such that $E$ is a subset of the set of unordered pairs of elements of $V$. The set $V=V(G)$ is called the vertex set of $G$ and its elements are called the vertices of $G$. The set $E=E(G)$ is called the edge set of $G$ and 
its elements are called the edges of $G$. The vertex set is always assumed to be nonempty but the edge set may be empty. If both the vertex set and the edge set are finite then the graph is said to be a finite graph.

An edge $e$ which is an unordered pair of vertices $x$ and $y$ is written as $e=x y=y x$ and $e$ is said to join $x$ and $y$. The vertices $x$ and $y$ joined by $e$ are called the end vertices of $e$ and $x$ and $y$ are also said to be incident with $e$.

A subgraph of $G$ is a graph $H$ such that $V(H) \subseteq V(G), E(H) \subseteq E(G)$. A spanning subgraph of $G$ is a subgraph $H$ of $G$ such that $V(H)=V(G)$.

A walk $W=x_{0} e_{1} x_{1} e_{2} x_{2} \cdots x_{N-1} e_{N} x_{N}$ is an alternating sequence of vertices and edges, where $e_{i}=x_{i-1} x_{i}, 1 \leq i \leq N$. The vertices $x_{0}$ and $x_{N}$ are the origin and terminus of $W$ and $x_{1}, x_{2}, \ldots, x_{N-1}$ are the internal vertices of $W$. The number $N$, is said to be the length of $W$.

A path is a walk with distinct vertices. A graph is connected if for every pair $\{x, y\}$ of distinct vertices there is a path from $x$ to $y$. A maximal connected subgraph is called a component of the graph.

If a walk $W=x_{0} e_{1} x_{1} e_{2} x_{2} \cdots x_{N-1} e_{N} x_{N}$ is such that $N \geq 3, x_{0}=x_{N}$ and the vertices $x_{i}, 0<i<N$, are distinct from each other and $x_{0}$ then $W$ is said to be a cycle. A graph without any cycles is a forest; a connected graph without any cycles is a tree. A spanning tree of a graph is a spanning subgraph that is a tree.

The following results are assumed in the next section. For details, see [4].

A. Every vertex of a graph belongs to a unique component.

B. In a tree any two vertices are joined by a unique path.

C. Every connected graph contains a spanning tree.

\subsection{Marked graphs.}

Definition. Let $G$ be a graph. If to each vertex in $G$ one of $(0),(+)$ or $(-)$ is attached then $G$ is said to be a marked graph.

Remark. For our applications we described in $\S 2$ how to associate a graph to a finite direct sum of weighted bilateral shifts and how to attach $(0),(+)$ or $(-)$ to the vertices of the graph. The above definition of a marked graph is more general than the one introduced by Lowell W. Beineke and Frank Harary in [ 2 and 3], as well as the one we have given in $\S 2$.

In this section we show that if a component $C$ of a marked graph $G$ has (a) an equal number of $(+)$ and $(-)$ vertices or (b) at least one (0) vertex, then there exists an admissible sequence of edges (defined later) in $C$ such that every vertex of $C$ except possibly one of the $(0)$ vertices occurs an odd number of times as an end vertex of edges in the sequence.

Lemma 3.2.1. Let $G$ be a marked graph and $K$ a spanning tree of a component $C$ of $G$. If $C$ has the same number of $(+)$ and $(-)$ vertices, say $l$, and no (0) vertex, then there exists a sequence $\left\{P_{1}, P_{2}, \ldots, P_{l}\right\}$ of paths in $K$ satisfying the following:

(a) the origin and terminus of each path have opposite signs and neither of them is present in any of the previous paths in the sequence, and

(b) every internal vertex of each path belongs to at least one of the previous paths in the sequence. 
Proof. Suppose $l=1$. Because $K$ is connected there exists an edge joining a $(+)$ vertex to a $(-)$ vertex. This edge is defined to be the path $P_{1}$.

Suppose $l>1$. Assume that for some $r$ such that $1 \leq r<l$ the paths $P_{1}, P_{2}, \ldots, P_{r}$ have been constructed satisfying the two conditions stated in the lemma. Because $r<l$ there is a $(+)$ vertex and a $(-)$ vertex in $K$, not present in any of the paths $P_{1}, P_{2}, \ldots, P_{r}$. Let $Q$ be the unique path in $K$ connecting these two vertices. Suppose $\mathscr{L}$ is the set of lengths of the paths contained in $Q$ whose origin and terminus are of opposite signs and are not in $P_{1}, P_{2}, \ldots, P_{r}$. This set is nonempty because $Q$ is such a path. Let $s$ be the minimum of the set $\mathscr{L}$. There exists a path $Q^{*}$ of length $s$ such that its origin and terminus are of opposite signs and are not present in $P_{1}, P_{2}, \ldots, P_{r}$. Every internal vertex, if any, of $Q^{*}$ must be in one of $P_{1}, P_{2}, \ldots, P_{r}$. If not, it will contradict the fact that $s$ is the minimum of the set $\mathscr{L}$. Now, $Q^{*}$ is defined to be the path $P_{r+1}$. The paths $P_{1}, P_{2}, \ldots, P_{l}$, thus, are defined recursively.

Lemma 3.2.2. Let $G$ be a marked graph and $K$ a spanning tree of a component $C$ of $G$. If $C$ has exactly one (0) vertex then there exists a sequence $\left\{P_{1}, P_{2}, \ldots, P_{l}\right\}$ of paths in $K$ satisfying the following:

(a) each path has its origin at the (0) vertex; the terminus of each path is $a(+)$ vertex or a (-) vertex that is not present in any of the previous paths in the sequence, and

(b) every internal vertex of each path belongs to at least one of the previous paths in the sequence.

Proof. In the first stage of the construction of the sequence of paths the $r$ edges incident with the $(0)$ vertex are defined as the first $r$ paths $P_{1}, P_{2}, \ldots, P_{r}$.

Suppose that the paths $P_{1}, P_{2}, \ldots, P_{s}$ for some $s \geq r$ have been constructed in the first $j$ stages, such that each path satisfies the two conditions stated in the lemma. In the $(j+1)$ th stage the terminal vertices of the paths constructed in the $j$ th stage are examined. Suppose that the terminal vertex of a path $P_{k}$ is joined by an edge $e$ to a vertex not belonging to any of the paths $P_{1}, P_{2}, \ldots, P_{s}$. Now, this edge $e$ is joined to $P_{k}$ to give a path in the $(j+1)$ th stage. The paths in the $(j+1)$ th stage are thus defined. Because $K$ is a finite tree the construction stops in a finite number of stages. Note that the number of paths $l$ is the number of edges in the spanning tree $K$.

Definition. An ordered sequence of edges in a marked graph is said to be admissible if every edge $e$ in the sequence satisfies one of the following:

(i) $e$ is incident with a $(0)$ vertex,

(ii) the end vertices of $e$ are of opposite signs and both have occurred as end vertices of edges prior to $e$ in the sequence of edges an even number of times or both an odd number of times,

(iii) the end vertices of $e$ are of the same sign and one has occurred as end vertex of edges prior to $e$ in the sequence of edges an even number of times and the other an odd number of times.

Remark. In the next two lemmas we show how to augment a given admissible sequence of edges.

Lemma 3.2.3. Let $G$ be a marked graph and $K$ a spanning tree of a component $C$ of $G$. Let an admissible sequence of edges $\left\{e_{1}, e_{2}, \ldots, e_{r}\right\}$ in $C$ be given. 
Let $P$ be a path in $K$ having only $(+)$ and $(-)$ vertices and that (1) its initial and terminal vertices are of opposite signs and have occurred an even number of times as end vertices of edges in the given sequence of edges and (2) its internal vertices have occurred an odd number of times as end vertices of edges in the given sequence of edges. Then, the edges of $P$ can be ordered so that the result of augmenting the given sequence by them still gives an admissible sequence of edges.

Proof. The proof is by induction on the length of $P$, denoted by $L(P)$.

Suppose $L(P)=1$, then from the hypothesis $P$ is an edge joining a $(+)$ vertex to a $(-)$ vertex and both vertices have occurred an even number of times as end vertices of edges in the given sequence of edges. Therefore $P$ can be labelled $e_{r+1}$.

Suppose the conclusion of the lemma is true when $L(P) \leq N$. For a path $P$ with $L(P)=N+1$ there are three cases to be considered.

Case 1. The initial $(+)$ vertex, denoted by $m$, is joined to an internal $(+)$ vertex, denoted by $m^{\prime}$. By the hypothesis of the lemma $m$ has occurred as an end vertex of edges in $\left\{e_{1}, e_{2}, \ldots, e_{r}\right\}$ an even number of times and $m^{\prime}$ an odd number of times. Hence the edge $\mathrm{mm}^{\prime}$ can be labelled $e_{r+1}$. Now, the path $P^{\prime}$, contained in $P$, from $m^{\prime}$ to the terminal vertex of $P$ satisfies the hypothesis of this lemma because $m^{\prime}$ has now occurred as an end vertex of edges in the sequence $\left\{e_{1}, e_{2}, \ldots, e_{r}, e_{r+1}\right\}$ an even number of times. Using the induction hypothesis on $P^{\prime}$ the construction can be completed.

Case 2. If in $P$, the initial $(-)$ vertex is joined to an internal (-) vertex, then the construction proceeds similarly to Case 1 .

Case 3. If in $P$, the initial $(+)$ vertex is joined to an internal $(-)$ vertex and the terminal $(-)$ vertex is joined to an internal $(+)$ vertex then there is an edge joining an internal $(-)$ vertex, denoted by $n^{\prime}$, to an internal $(+)$ vertex, denoted by $m^{\prime}$. By the hypothesis of the lemma both $m^{\prime}$ and $n^{\prime}$ have occurred as end vertices of edges in $\left\{e_{1}, e_{2}, \ldots, e_{r}\right\}$ an odd number of times. Therefore, the edge $n^{\prime} m^{\prime}$ can be labelled $e_{r+1}$.

Now, define two disjoint paths $P^{\prime}$ and $P^{\prime \prime}$ contained in $P$, where $P^{\prime}$ is the path from the initial $(+)$ vertex of $P$ to the $(-)$ vertex $n^{\prime}$ and $P^{\prime \prime}$ is the path from the $(+)$ vertex $m^{\prime}$ to the terminal $(-)$ vertex of $P$. Because $n^{\prime}$ and $m^{\prime}$ have now occurred as end vertices of edges in the sequence of edges $\left\{e_{1}, e_{2}, \ldots, e_{r}, e_{r+1}\right\}$ an even number of times both $P^{\prime}$ and $P^{\prime \prime}$ satisfy the hypothesis of this lemma. Since both $P^{\prime}$ and $P^{\prime \prime}$ have lengths less than or equal to $N$, the construction can be completed by the induction hypothesis.

This concludes the proof of the lemma.

Lemma 3.2.4. Let $G$ be a marked graph and $K$ a spanning tree of a component $C$ of $G$ having exactly one (0) vertex. Let an admissible sequence of edges $\left\{e_{1}, e_{2}, \ldots, e_{r}\right\}$ in $C$ be given. If $P$ is a path in $K$ such that (1) its origin is the (0) vertex and its terminus is a (+) or (-) vertex that has occurred an even number of times in the given sequence of edges and (2) its internal vertices are $(+)$ or (-) vertices that have occurred an odd number of times in the given sequence of edges, then the edges of $P$ can be ordered so that the result of augmenting the given sequence by them still gives an admissible sequence of edges.

Proof. The conclusion of the lemma is shown below for the six possible cases of $P$. 
Case 1. Suppose the (0) vertex is joined to a $(+)$ vertex, denoted by $m$, and the terminal vertex is a $(-)$ vertex. The edge incident with $(0)$ vertex and $m$ can be labelled $e_{r+1}$. Now, let $P^{\prime}$ be the path contained in $P$ from $m$ to the terminal vertex of $P$. Because $m$ has now occurred even number of times in the sequence $\left\{e_{1}, e_{2}, \ldots, e_{r}, e_{r+1}\right\}$, Lemma 3.2.3 applies to $P^{\prime}$. This completes the construction.

Case 2. Suppose the $(0)$ vertex is joined to a $(-)$ vertex and the terminal vertex of $P$ is a $(+)$ vertex. This case is similar to Case 1 .

Case 3. Suppose the $(0)$ vertex is joined to a $(+)$ vertex and all the internal vertices and the terminus are $(+)$ vertices. Then the terminal $(+)$ vertex has occurred an even number of times and the internal $(+)$ vertex adjacent to the terminus has occurred an odd number of times. So this edge can be labelled $e_{r+1}$. The edges of $P$ can now be added to the admissible sequence in the reverse order.

Case 4. The case where all the vertices of $P$, other than the $(0)$ vertex, are (-) vertices is similar to Case 3.

Case 5. Suppose the $(0)$ vertex is joined to an internal $(+)$ vertex, denoted by $m^{\prime}$, the terminal vertex is also a $(+)$ vertex, denoted by $m$, and that there are some internal $(-)$ vertices, unlike Case 3 . Then, there is an internal $(+)$ vertex, denoted by $m^{\prime \prime}$, and an internal $(-)$ vertex, denoted by $n$, both incident on an edge and such that all the vertices between $m^{\prime}$ and $m^{\prime \prime}$ are $(+)$ vertices. By the hypothesis of the lemma both $m^{\prime \prime}$ and $n$ have occurred an odd number of times and therefore the edge $m^{\prime \prime} n$ can be labelled $e_{r+1}$. Now, both $m^{\prime \prime}$ and $n$ have occurred an even number of times in $\left\{e_{1}, e_{2}, \ldots, e_{r}, e_{r+1}\right\}$. If two disjoint paths $P^{\prime}$ and $P^{\prime \prime}$ contained in $P$ are considered, where $P^{\prime}$ is the path from the $(0)$ vertex to $m^{\prime \prime}$ and $P^{\prime \prime}$ is the path from $n$ to $m$ then $P^{\prime}$ satisfies the conditions of Case 3 of this lemma and $P^{\prime \prime}$ satisfies the hypothesis of Lemma 3.2.3. This completes the construction in Case 5.

Case 6. If in $P$ the (0) vertex is joined to an internal (-) vertex, if the terminal vertex is also a $(-)$ vertex and if there are some internal $(+)$ vertices, the construction proceeds as in Case 5.

This completes the proof of the lemma.

Theorem 3.2.1. let $G$ be a marked graph and $C$ a component of $G$. Suppose $C$ has either (a) an equal number of $(+)$ and $(-)$ vertices or $(b)$ at least one $(0)$ vertex, then there exists an admissible sequence of edges such that each vertex except possibly a pre-assigned (0) vertex occurs an odd number of times as an end vertex of edges in the sequence.

Proof. If $C$ has an equal number of $(+)$ and $(-)$ vertices and no $(0)$ vertex then the conclusion follows from Lemmas 3.2.1 and 3.2.3.

Suppose $C$ has more than one $(0)$ vertex. Then assign a $(+)$ to all the (0) vertices except one. Then using Lemmas 3.2.2 and 3.2.4 there is an admissible sequence of edges. After changing back from $(+)$ to $(0)$ it is clear that the sequence of edges is still admissible. This concludes the proof of the theorem. 


\section{Proof of THE SUfFICIENCY}

Given a component $C$ of the marked graph $G$ associated with $T$ satisfying one of the two conditions of the main theorem we describe a procedure to construct a projection $P_{n}(C)$ of finite rank associated with $C$ for each given positive integer $n$. If $T_{C}$ denotes the direct sum of the ends corresponding to the vertices in $C$ it will be shown that $\lim _{n \rightarrow \infty}\left\|P_{n}(C) T_{C}-T_{C} P_{n}(C)\right\|=0$. Moreover, if $\mathscr{H}_{C}$ denotes the direct sum of the spaces on which the ends of $C$ are defined then we show that $P_{n}(C)$ converges to the identity on $\mathscr{H}_{C}$. Therefore we conclude that $T_{C}$ is in $(Q D)$. Now, it follows that $T$ is in $(Q D)$ because $T$ is the finite direct sum of $T_{C}$ as $C$ varies over the components of $G$.

4.1. Basic assumptions. If the component $C$ of the marked graph $G$ is not a tree we replace $C$ by one of its spanning trees. This leads to no loss of generality in our theorem.

If more than one $(0)$ vertex is present in $C$, then assign a $(+)$ to all the $(0)$ vertices except one. Again this leads to no loss of generality.

The weight sequence corresponding to the $(0)$ vertex has a subsequence with limit zero. By changing these weights to zero we obtain a compact perturbation of $T_{C}$. We again denote it by $T_{C}$. The distance between two successive zeros on this end is assumed to approach infinity.

From Theorem 3.2.1 there is an admissible sequence of edges in $C$ such that each vertex except possibly the $(0)$ vertex occurs an odd number of times as an end vertex of edges in the sequence. From the proof of Theorem 3.2.1 we obtain a sequence of edges in a spanning tree of $C$. This admissible sequence of edges will enable us to construct the projection $P_{n}(C)$.

4.2. Edge operators. In the construction of the projection $P_{n}(C)$ the 'edge operators' play a central role. We associate an 'edge operator' to each edge in the admissible sequence of edges following certain rules to be described later. We begin with the following definitions.

Definition. An operator $A$ is a [0-1] diagonal on an end $T_{a}$ if its domain is the span of $\left\{e_{r}^{a}, e_{r+1}^{a}, \ldots, e_{r+n}^{a}\right\}$ and $A e_{r+p}^{a}=\left(\frac{p}{n}\right) e_{r+p}^{a}$ for $0 \leq p \leq n$, where $n$ is a given positive integer and $r$ is an integer.

Definition. An operator $D$ is a $[1-0]$ diagonal on an end $T_{b}$ if its domain is the span of $\left\{e_{s}^{b}, e_{s+1}^{b}, \ldots, e_{s+n}^{b}\right\}$ and $D e_{s+p}^{b}=\left(\frac{n-p}{n}\right) e_{s+p}^{b}$ for $0 \leq p \leq n$, where $n$ is a given positive integer and $s$ is an integer.

Definition. Let $e$ be an edge in the marked graph $G$ joining the ends $T_{a}$ and $T_{b}$. An edge operator $E$ associated with $e$ is given by the $2 \times 2$ operator matrix

$$
E=\left[\begin{array}{cc}
A & B \\
B^{*} & D
\end{array}\right]
$$

where the entries satisfy the following:

(i) $A$ is a $[0-1]$ diagonal on the end $T_{a}$,

(ii) $D$ is a $[1-0]$ diagonal on the end $T_{b}$,

(iii) $B=\left(A-A^{2}\right)^{1 / 2} V^{*}$ where $V$ is the partial isometry from the domain of $A$ to the domain of $D$ defined by $V e_{r+p}^{a}=e_{s+p}^{b}$ for $0 \leq p \leq n$. 
Thus $E$ is defined on the domain of $(A \oplus D)$ contained in $\mathscr{H}_{e}$, the direct sum of the spaces corresponding to the ends in $e$.

Lemma 4.2.1. If $E$ is an edge operator then (a) $E^{*}=E$ and (b) $E^{2}=E$.

The proof of this lemma is left as an exercise.

4.3. Construction of the edge operators. We will construct a sequence of edge operators corresponding to our admissible sequence of edges. Suppose a vertex is incident on several edges; then for each edge a [0-1] or a [1-0] diagonal will be constructed on distinct blocks of the end. When constructing the diagonals on each end the following three rules will be applied.

(1) On the end corresponding to the (0) vertex each diagonal is constructed between two zero weights.

(2) On a positive end the diagonals are constructed from left to right without overlaps. They alternate between $[1-0]$ and $[0-1]$ with the first and the last being a $[1-0]$ diagonal.

(3) On a negative end the diagonals are constructed from right to left without overlaps. They alternate between $[1-0]$ and $[0-1]$ with the first and the last being a $[0-1]$ diagonal.

We first explain this construction in the following examples.

Example 1. Let $C$ be given by

$$
\text { 0(0) } \quad 2(+)
$$

An admissible sequence of edges is $\left\{e_{01}, e_{12}, e_{01}\right\}$. A pictorial description of edge operators on $C$ is given below.

$$
\begin{aligned}
& \overbrace{\overbrace{}^{V_{1}}}^{V_{3}} \\
& . . . .[0-1] \ldots \bullet \ldots \overbrace{[0-1] \ldots \bullet \ldots 0(0)}^{1(+) \ldots[1-0] \ldots[0-1] \ldots[1-0] \ldots} \\
& \downarrow V_{2} \\
& 2(+) \ldots \ldots \ldots \ldots[1-0] \ldots \ldots
\end{aligned}
$$

In the picture $V_{1}, V_{2}$ and $V_{3}$ are partial isometries and $\bullet$ denote the zero weights.

Example 2. If $C$ is given by

$$
\stackrel{2}{1(-)} \quad \mathbf{3}(+) \quad 4(+)
$$

An admissible sequence of edges is $\left\{e_{23}, e_{34}, e_{12}, e_{23}\right\}$. The edge operators are given below.

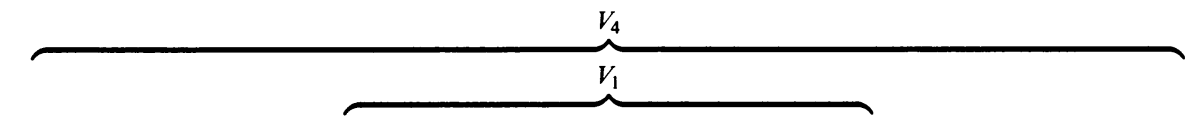

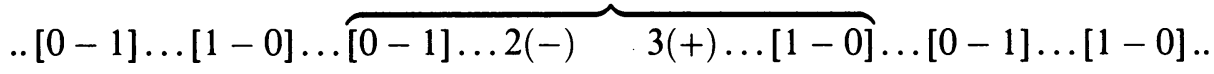

$$
\begin{aligned}
& \uparrow V_{3} \quad \downarrow V_{2} \\
& \text {....... }[0-1] \ldots \ldots \ldots \ldots 1(-) \quad 4(+) \ldots \ldots \ldots[1-0] \ldots \ldots
\end{aligned}
$$

The partial isometries in the four edge operators are labelled as $V_{1}, V_{2}, V_{3}$ and $V_{4}$. 
In general, consider the admissible sequence of edges given by Theorem 3.2.1. Suppose $e=a b$ is an edge in the sequence. If $T_{a}$ and $T_{b}$ are the corresponding block balanced ends of $T$ with weight sequences $\left\{w_{j}^{a}\right\}$ and $\left\{w_{i}^{b}\right\}$ then for the given positive integer $n$ there exist integers $r$ and $s$ such that $\left|w_{r+p}^{a}-w_{s+p}^{b}\right|<\frac{1}{n}$ for $0 \leq p \leq n$. We use such positions to construct the edge operator $E$ associated with $e$.

There are three cases in the construction of $E$.

Case 1. If the edge $e$ is incident with the (0) vertex, then the other vertex is a $(+)$ or a $(-)$. Suppose it is a (+) vertex. Also, suppose that the positive end has an odd number of diagonals already constructed. Then $E$ has a $[0-1]$ diagonal on the positive end and a $[1-0]$ diagonal on the end corresponding to the $(0)$ vertex. If the positive end has an even number of diagonals then construct $E$ with a [0-1] diagonal on the end corresponding to the $(0)$ vertex and a $[1-0]$ diagonal on the positive end. When a $(-)$ vertex is incident on $e$ the construction of $E$ is similar.

Case 2. If the end vertices of $e$ are of opposite signs and both have occurred an even number of times prior to $e$ in the sequence or both an odd number of times, then the construction of $E$ is done as follows. If we assume that both ends have an even number of diagonals on them, then for $E$, a [0-1] diagonal is constructed on the negative end and a [ $1-0]$ diagonal is constructed on the positive end. The construction is handled the opposite way if the diagonals are odd in number.

Case 3. If the end vertices of $e$ are of the same sign and one has occurred an even number of times prior to $e$ in the sequence and the other an odd number of times, then there are two possibilities to consider. Suppose both are positive ends. Then for $E$, a $[0-1]$ diagonal is constructed on the end with odd number of diagonals already constructed and a [1-0] diagonal is constructed on the end with even number of diagonals. When the ends are both negative the construction is similar.

To summarize the construction of edge operators we say the following. If a component $C$ has either an equal number of $(+)$ and $(-)$ vertices or one (0) vertex then there exists an admissible sequence of edges in $C$ such that each vertex occurs an odd number of times in the sequence except possibly the $(0)$ vertex. For each edge in the sequence we construct an edge operator such that the number of diagonal operators on each end, except possibly the end corresponding to the $(0)$ vertex, is odd. We call these edge operators the admissible sequence of edge operators.

4.4. Construction of projections. We define $P_{n}(C)$ to be the direct sum of the edge operators in the admissible sequence of edge operators constructed in 4.3 and an operator $R$ defined on the orthogonal complement in $\mathscr{H}_{C}$ of the domains of these edge operators.

$R$ is a diagonal operator with entries 0 and 1 . On the end corresponding to a $(0),(+)$ or $(-)$ vertex $R$ is 0 between consecutive [1-0] and [0-1] diagonals and 1 between consecutive $[0-1]$ and $[1-0]$ diagonals; $R$ is 1 to the left or right of the first diagonal if the end is a $(+)$ or a $(-)$ and 0 to the 
right or left of the last diagonal. We show these in the following diagrams.

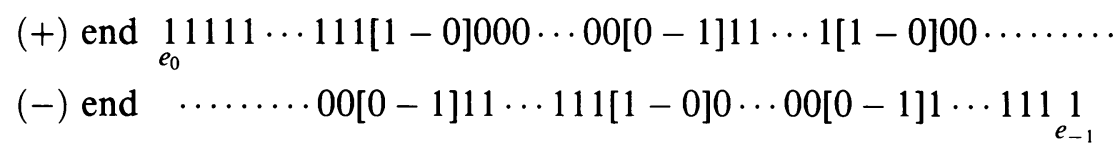

In addition to the above, on the end corresponding to the $(0)$ vertex define $R$ as follows: If there are consecutive [0-1] diagonals, for example, and one of them is between two successive zero weights at $c$ and $d$ then $R$ is 0 from $(c+1)$ to the diagonal and 1 from the diagonal to $d$. For example,

$\underset{e_{0}}{11 \cdots 11} \bullet 00[0-1] 111 \cdots 11[1-0] 00 \cdots 0[0-1] 111 \cdots 1 \underbrace{}_{c} 100 \cdots 0[0-1] 1100 \cdots \cdots$

where $\bullet$ are the zero weights of the end; $R$ is similar on the negative end corresponding to the $(0)$ vertex. A similar definition is given when the consecutive diagonals are $[1-0]$.

Lemma 4.4.1. $P_{n}(C)$ is a finite rank projection.

Proof. It is clear that $P_{n}(C)$ has finite rank. From Lemma 4.2.1 every edge operator is a projection on its domain. From the definition of $R$ the conditions $R^{*}=R$ and $R^{2}=R$ follow easily.

\subsection{Conclusion of the proof.}

Lemma 4.5.1. If the choices in the definition of $P_{n}(C)$ are made appropriately, then $P_{n}(C) \rightarrow I$ strongly on $\mathscr{H}_{C}$.

Proof. For a fixed $i$ and a given positive integer $n>|i|$ the first diagonal on the end can be constructed to the right of $e_{i}$ if $i>0$ and to the left of $e_{i}$ if $i<0$. Therefore $P_{n}(C) e_{i}=e_{i}$. This shows that we can achieve $P_{n}(C) \rightarrow I$ on $\mathscr{H}_{C}$ strongly.

In the next three lemmas we show that $\left\|P_{n}(C) T_{C}-T_{C} P_{n}(C)\right\| \rightarrow 0$.

Lemma 4.5.2. The restriction of $P_{n}(C) T_{C}-T_{C} P_{n}(C)$ to the domain of $R$ is zero.

Proof. From the definition of $R$ when both $e_{i}$ and $e_{i+1}$ are eigenvectors of $R$ corresponding to the eigenvalue 0 or eigenvalue 1 we easily see that $R T_{C} e_{i}$ $T_{C} R e_{i}=0$. If $e_{i}$ is in the space corresponding to the (0) vertex such that $R e_{i}=e_{i}$ and $R e_{i+1}=0$ then from the definition of $R$ the weight $w_{i}$ is zero. Therefore $R T_{C} e_{i}-T_{C} R e_{i}=0$. To complete the proof we note that when $e_{i}$ and $e_{i+1}$ are at the boundaries of $R$ and the diagonals, the eigenvalue of $P_{n}(C)$ is the same. This shows that $P_{n}(C) T_{C}-T_{C} P_{n}(C)$ restricted to the domain of $R$ is zero.

Suppose $T$ has a polar decomposition $\mathscr{W}_{0}$, then $\mathscr{W}$ is the direct sum of the unweighted bilateral shift $W$ ( $k$ times) and $\mathscr{D}_{0}$ is the direct sum of $k$ diagonal operators, each based on the weight sequence of $T_{i}$ for $1 \leq i \leq k$.

Lemma 4.5.3. If $S_{n}$ is the restriction of $P_{n}(C) \mathscr{D}_{0}-\mathscr{D}_{0} P_{n}(C)$ to the domain of the sequence of edge operators, then $\left\|S_{n}\right\|<\frac{1}{n}$.

Proof. Suppose $E=\left[\begin{array}{cc}A & B \\ B^{*} & D\end{array}\right]$ is an edge operator. Then the domain of $E$ is a reducing subspace of $P_{n}(C) \mathscr{D}_{0}-\mathscr{D}_{0} P_{n}(C)$. If $\mathscr{D}_{0}$ is restricted to the domain of 
$E$, then it is a direct sum of the diagonal operators $D_{a}$ and $D_{b}$ corresponding to the two ends $T_{a}$ and $T_{b}$. To estimate the norm of $S_{n}$ let us suppose $e_{i}$ is in the domain of $A$. Then $i=r+p$ for an integer $r$ and $0 \leq p \leq n$. In this case $A D_{a} e_{r+p}^{a}-D_{a} A e_{r+p}^{a}=0$. We now compute $\left(B^{*} \mathscr{D}_{0}-\mathscr{D}_{0} B^{*}\right) e_{r+p}^{a}$ for $0 \leq p \leq n$.

$$
\begin{aligned}
\left(B^{*} \mathscr{D}_{0}-\mathscr{D}_{0} B^{*}\right) e_{r+p}^{a} & =V\left(A-A^{2}\right)^{1 / 2} D_{a} e_{r+p}^{a}-D_{b} V\left(A-A^{2}\right)^{1 / 2} e_{r+p}^{a} \\
& =\left[\left(\frac{p}{n}\right)-\left(\frac{p}{n}\right)^{2}\right]^{1 / 2}\left(w_{r+p}^{a}-w_{s+p}^{b}\right) e_{s+p}^{b} .
\end{aligned}
$$

Therefore,

for $0 \leq p \leq n$.

$$
\left\|\left(B^{*} \mathscr{D}_{0}-\mathscr{D}_{0} B^{*}\right) e_{r+p}^{a}\right\| \leq\left|w_{r+p}^{a}-w_{s+p}^{b}\right|<\frac{1}{n}
$$

Because the images of $e_{r+p}^{a}$ for $0 \leq p \leq n$ are orthogonal under $A$ and $B^{*}$ we conclude that $A \mathscr{D}_{0}=\mathscr{D}_{0} A$ and $\left\|B^{*} \mathscr{D}_{0}-\mathscr{D}_{0} B^{*}\right\|<\frac{1}{n}$. A similar estimate is obtained when $e_{i}$ is in the domain of $D$. Therefore we see that $\left\|S_{n}\right\|<\frac{1}{n}$.

Lemma 4.5.4. If $L_{n}$ is the restriction of $P_{n}(C) \mathscr{W}-\mathscr{W} P_{n}(C)$ to the domain of the sequence of edge operators, then $\left\|L_{n}\right\|<2 / \sqrt{n}$.

Proof. Suppose $E=\left[\begin{array}{c}A \\ B^{*}\end{array}\right]$ ]

If $e_{i}$ and $e_{i+1}$ are both in the domain of $A$, then $i=r+p$ for some integer $r$ and $0 \leq p \leq n-1$. From the definition of $A$ we get

$$
\left\|(A \mathscr{W}-\mathscr{W} A) e_{r+p}^{a}\right\|=\left\|\left[\left(\frac{p+1}{n}\right)-\left(\frac{p}{n}\right)\right] e_{r+p+1}^{a}\right\|=\frac{1}{n} .
$$

We now compute $\left\|\left(B^{*} \mathscr{W}-\mathscr{W} B^{*}\right) e_{r+p}^{a}\right\|$.

$$
\begin{aligned}
B^{*} \mathscr{W} e_{r+p}^{a} & =V\left(A-A^{2}\right)^{1 / 2} \mathscr{W} e_{r+p}^{a}=V\left(A-A^{2}\right)^{1 / 2} e_{r+p+1}^{a} \\
& =\left[\left(\frac{p+1}{n}\right)-\left(\frac{p+1}{n}\right)^{2}\right]^{1 / 2} e_{s+p+1}^{b} \\
& =\left[\left(\frac{p+1}{n}\right)\left(\frac{n-p-1}{n}\right)\right]^{1 / 2} e_{s+p+1}^{b} \\
& =\frac{\sqrt{(p+1)(n-p-1)}}{n} e_{s+p+1}^{b} . \\
\mathscr{W} B^{*} e_{r+p}^{a} & =\mathscr{W} V\left(A-A^{2}\right)^{1 / 2} e_{r+p}^{a} \\
& =\mathscr{W} V\left[\left(\frac{p}{n}\right)-\left(\frac{p}{n}\right)^{2}\right]^{1 / 2} e_{r+p}^{a} \\
& =\left[\left(\frac{p}{n}\right)-\left(\frac{p}{n}\right)^{2}\right]^{1 / 2} e_{s+p+1}^{b} \\
& =\frac{\sqrt{p(n-p)}}{n} e_{s+p+1}^{b} .
\end{aligned}
$$

Therefore

$$
\left\|\left(B^{*} \mathscr{W}-\mathscr{W} B^{*}\right) e_{r+p}^{a}\right\|=\left|\frac{\sqrt{(p+1)(n-p-1)}}{n}-\frac{\sqrt{p(n-p)}}{n}\right|<\frac{1}{\sqrt{n}} .
$$


Because the images of $e_{r+p}^{a}$ for $0 \leq p \leq n$ are orthogonal under $A$ and $B^{*}$ we conclude that $\|A \mathscr{W}-\mathscr{W} A\|=1 / n$ and $\left\|B^{*} \mathscr{W}-\mathscr{W} B^{*}\right\|<1 / \sqrt{n}$. A similar estimate is obtained when both $e_{i}$ and $e_{i+1}$ are in the domain of $D$. Now for $e_{i}$ and $e_{i+1}$ in the boundaries of $R$ and one of the diagonals $A$ or $D$ it can easily be seen that $L_{n} e_{i}=0$. Therefore we see that $\left\|L_{n}\right\|<1 / n+1 / \sqrt{n}<2 / \sqrt{n}$.

When $P_{n}(C) T_{C}-T_{C} P_{n}(C)$ is restricted to the domain of the sequence of edge operators we find that

$$
\left\|P_{n}(C) T_{C}-T_{C} P_{n}(C)\right\| \leq\|\mathscr{W}\|\left\|S_{n}\right\|+\left\|L_{n}\right\|\left\|\mathscr{D}_{0}\right\|
$$

From the above lemmas we see that $\left\|P_{n}(C) T_{C}-T_{C} P_{n}(C)\right\| \rightarrow 0$ as $n \rightarrow \infty$.

Therefore we conclude that $\left\{P_{n}(C)\right\}_{1}^{\infty}$ is a sequence of finite rank projections that implements the quasidiagonality of $T_{C}$. Therefore, if each component of $G$ satisfies one of the two conditions of the main theorem then $T$ is quasidiagonal. This completes the proof of the sufficiency.

\section{Proof OF THE NECESSITY}

Suppose one of the components in the marked graph $G$ associated with $T$ fails to satisfy either of the conditions of the theorem. We prove that $T$ is not quasidiagonal. In fact we show that the $C^{*}$-algebra generated by $T$ contains $(J \oplus 0)+K$ where $J$ is a Fredholm operator with nonzero index and $K$ is compact. Moreover the Fredholm operator $J$ is shown to be of the form $U \oplus \cdots \oplus U \oplus U^{*} \oplus \cdots \oplus U^{*}$ where the unilateral shift $U$ and its adjoint $U^{*}$ occur an unequal number of times.

5.1. Some preliminaries. Let $\mathscr{W} \mathscr{D}_{0}$ be the polar decomposition of $T$ where $\mathscr{W}$ is the direct sum of the unweighted bilateral shift $W$ ( $k$ times) and $\mathscr{D}_{0}$ is the direct sum of $k$ diagonal operators.

Let

$$
\mathscr{E}=\underbrace{l^{\infty}(\mathbf{Z}) \oplus \cdots \oplus l^{\infty}(\mathbf{Z})}_{k \text { times }} .
$$

Then $\mathscr{E}$ is isometrically $*$-isomorphic to $C(X)$ where

$$
X=\underbrace{\beta(\mathbf{Z}) \amalg \beta(\mathbf{Z}) \amalg \cdots \amalg \beta(\mathbf{Z})}_{k \text { times }},
$$

where $\amalg$ denotes a disjoint union and $\beta(Z)$ is the Stone-Čech compactification of $\mathbf{Z}$. If $\mathscr{D}$ is in $\mathscr{E}$ then $D_{i}$, the $i$ th component of $\mathscr{D}$, belongs to $l^{\infty}(\mathbf{Z})$. If

$$
\mathscr{H}_{k}=\underbrace{\mathscr{H} \oplus \mathscr{H} \oplus \cdots \oplus \mathscr{H}}_{k \text { times }}
$$

then the obvious representation $\pi: \mathscr{E} \rightarrow \mathscr{B}\left(\mathscr{H}_{k}\right)$ maps $D_{i}$ onto a diagonal operator on $\mathscr{H}$ also denoted by $D_{i}$.

Suppose

$$
\mathbf{Z}_{k}=\underbrace{\mathbf{Z} \amalg \mathbf{Z} \amalg \cdots \amalg \mathbf{Z}}_{k \text { times }} ;
$$

then denote by $(n, i)$ the integer $n$ in the $i$ th copy of $\mathbf{Z}$. Moreover, $\mathbf{Z}_{k}$ will be regarded as a dense subset of $X$. 
5.2. An algebra $\mathscr{A}$ of diagonal operators. Let $\mathscr{A}$ be the collection of diagonal operators in $C^{*}(T)$, the $C^{*}$-algebra generated by $T$. Observe that $\mathscr{A}$ is a $C^{*}$ subalgebra of $\mathscr{E}$. Also, $\mathscr{A}$ can be identified with a $C^{*}$-subalgebra of $C(X)$, also denoted by $\mathscr{A}$.

Define $X_{0}=\{x \in X: f(x)=0$ for every $f$ in $\mathscr{A}\}$. Define an equivalence relation on $X$ by $x_{1} \sim x_{2}$ if $f\left(x_{1}\right)=f\left(x_{2}\right)$ for every $f$ in $\mathscr{A}$. Note that $X_{0}$ is an equivalence class. Denote by $p_{0}$ its image in the space $Y=X / \sim$. Using the Stone-Weierstrass theorem $\mathscr{A}$ is identified with $\left\{f \in C(Y): f\left(p_{0}\right)=0\right\}$.

Let Aut $\mathscr{E}$ denote the group of $*$-automorphisms of $\mathscr{E}$. Each $*$-automorphism of $\mathscr{E}$ induces a homeomorphism of $X$. If $\pi$ is the representation of $\mathscr{E}$ previously considered then the relation $\mathscr{W}^{*} \pi(\mathscr{D}) \mathscr{W}=\pi(\alpha(\mathscr{D}))$ for each $\mathscr{D}$ in $\mathscr{E}$ defines a $*$-automorphism $\alpha$ of $\mathscr{E}$. Let $\tau$ be the corresponding homeomorphism of $X$. Then, if $\mathscr{D}$ in $\mathscr{A}$ corresponds to $f$ in $C(X)$ then $\mathscr{W}^{*} \mathscr{D W}$ will correspond to $f \circ \tau$. It can easily be seen from the above relations that $\tau(n, i)=(n+1, i)$.

5.3. Some results about the algebra $\mathscr{A}$. Let $M\left(T, T^{*}\right)$ denote a monomial in $T$ and $T^{*}$ belonging to $C^{*}(T)$.

Let $\eta$ be the function defined on the collection of all monomials in $C^{*}(T)$ by $\eta(M)=($ the number of $T$ in $M)-\left(\right.$ number of $T^{*}$ in $\left.M\right)$.

Let $\mathscr{M}_{n}=\left\{M\left(T, T^{*}\right): \eta(M)=n\right\}$. It is clear that $\bigcup_{n \in \mathbf{Z}} \mathscr{M}_{n}$ generates $C^{*}(T)$.

Lemma 5.3.1. $\mathscr{A}$ is generated by $\mathscr{M}_{0}$.

Proof. It can easily be seen that $\mathscr{M}_{0}$ is closed under adjoints and multiplication. Therefore the algebra generated by $\mathscr{M}_{0}$ is its linear span. Since each member of $\mathscr{M}_{0}$ is a diagonal operator in $C^{*}(T)$ it belongs to $\mathscr{A}$. Therefore the norm closure of the linear span of $\mathscr{M}_{0}$ is contained in the $C^{*}$-subalgebra $\mathscr{A}$.

Note that if an operator $A: l^{2}(\mathbf{Z}) \rightarrow l^{2}(\mathbf{Z})$ has the matrix representation $\left[a_{i j}\right]$, then the operator norm of $\left[a_{i j}\right]$ is the same as $\|A\|$. It is given by

$$
\|A\|=\sup \left\{\left(\sum_{i}\left|\sum_{j} a_{i j} \xi_{j}\right|^{2}\right)^{1 / 2}: \sum\left|\xi_{j}\right|^{2} \leq 1\right\} .
$$

For the converse note that if $\mathscr{D}$ is a diagonal operator in $\mathscr{A}$ and $P$ is in the linear span of $\left\{\mathscr{M}_{n}: n\right.$ is a nonzero integer $\}$, then $\|\mathscr{D}-P\| \geq\|\mathscr{D}\|$. This can be seen from the matrix representation of the operators because the matrix of $P$ has only zeros along the main diagonal. Thus $\mathscr{A}$ is generated by $\mathscr{M}_{0}$.

We denote by $\Gamma: \mathscr{E} \rightarrow C(X)$ the Gelfand map.

Lemma 5.3.2. Let $T=\mathscr{W D}_{0}$ and $\Gamma\left(\mathscr{D}_{0}\right)=f_{0}$. Then $x$ is in $X_{0}$ if and only if $f_{0}(x)=f_{0}\left(\tau^{-1}(x)\right)=0$.

Proof. Since $\left|T^{*}\right|=\left|\mathscr{D}_{0} \mathscr{W}^{*}\right|=\left(\mathscr{W}_{0}^{2} \mathscr{W}^{*}\right)^{1 / 2}=\mathscr{W} \mathscr{D}_{0} \mathscr{W}^{*}$ it is clear that $\left|T^{*}\right|$ is in $\mathscr{A}$ and $\Gamma\left(\left|T^{*}\right|\right)=f_{0} \circ \tau^{-1}$. Therefore $f_{0}$ and $f_{0} \circ \tau^{-1}$ are in $\mathscr{A}$. If $x$ is in $X_{0}$ then $f_{0}(x)=0$ and $f_{0}\left(\tau^{-1}(x)\right)=0$ follow from the definition of $X_{0}$.

Conversely, assume that $\mathscr{D}$ is in $\mathscr{M}_{0}$ and $\Gamma(\mathscr{D})=f$. If $\mathscr{D}=T^{n_{1}} T^{* m_{1}} \ldots$ $T^{n_{k}} T^{* m_{k}}$ with $n_{i}, m_{i} \geq 0$ for $i=1, \ldots, k$ and $\sum_{i=1}^{k} n_{i}=\sum_{i=1}^{k} m_{i}$ then $\mathscr{D}^{*} \mathscr{D} \leq \gamma T^{*} T$ or $\gamma T T^{*}$ for some constant $\gamma$. Therefore $f_{0}(x)=0$ and 
$f_{0}\left(\tau^{-1}(x)\right)=0$ imply that $\Gamma\left(\mathscr{D}^{*} \mathscr{D}\right)(x)=|f|^{2}(x)=0$ for all such $f$. Hence $x$ is in $X_{0}$, by Lemma 5.3.1.

\section{Proposition 5.3.1.}

(1) If $x_{1} \sim x_{2}$ in $X$ then either (a) $f_{0}\left(x_{1}\right)=f_{0}\left(x_{2}\right)=0$ or (b) $\tau\left(x_{1}\right) \sim$ $\tau\left(x_{2}\right)$

(2) If $x_{1} \sim x_{2}$ in $X$ then either (c) $f_{0}\left(\tau^{-1}\left(x_{1}\right)\right)=f_{0}\left(\tau^{-1}\left(x_{2}\right)\right)=0$ or (d) $\tau^{-1}\left(x_{1}\right) \sim \tau^{-1}\left(x_{2}\right)$.

Proof. Let $\mathscr{D}$ be in $\mathscr{A}$ and $\Gamma(D)=f$. Then both $T \mathscr{D} T^{*}$ and $T^{*} \mathscr{D} T$ are in $\mathscr{A}$. Because $T \mathscr{D} T^{*}=\mathscr{W} \mathscr{D}_{0} \mathscr{D} \mathscr{D}_{0} \mathscr{W}^{*}$ it is clear that

$$
\Gamma\left(T \mathscr{D} T^{*}\right)=\left(f_{0} \circ \tau^{-1}\right)^{2}\left(f \circ \tau^{-1}\right) .
$$

Similarly, $T^{*} \mathscr{D} T=\mathscr{D}_{0} \mathscr{W} * \mathscr{D} \mathscr{W} \mathscr{D}_{0}$ implies that $\Gamma\left(T^{*} \mathscr{D} T\right)=\left(f_{0}\right)^{2}(f \circ \tau)$.

If $x_{1} \sim x_{2}$ then by the definition of the equivalence relation $f_{0}\left(x_{1}\right)=$ $f_{0}\left(x_{2}\right)$. From the above $\left(f_{0}\right)^{2}(f \circ \tau)$ is in $\mathscr{A}$. Therefore whenever $f$ is in $\mathscr{A},\left(f_{0}\right)^{2}\left(x_{1}\right)(f \circ \tau)\left(x_{1}\right)=\left(f_{0}\right)^{2}\left(x_{2}\right)(f \circ \tau)\left(x_{2}\right)$. If $f_{0}\left(x_{1}\right)=f_{0}\left(x_{2}\right) \neq 0$ then $f\left(\tau\left(x_{1}\right)\right)=f\left(\tau\left(x_{2}\right)\right)$ for each $f$ in $\mathscr{A}$. This proves (1) of the proposition.

If $x_{1} \sim x_{2}$ then $\left(f_{0} \circ \tau^{-1}\right)\left(x_{1}\right)=\left(f_{0} \circ \tau^{-1}\right)\left(x_{2}\right)$ because $f_{0} \circ \tau^{-1}$ is in $\mathscr{A}$. From the relation $\left(f_{0} \circ \tau^{-1}\right)^{2}\left(x_{1}\right)\left(f \circ \tau^{-1}\right)\left(x_{1}\right)=\left(f_{0} \circ \tau^{-1}\right)^{2}\left(x_{2}\right)\left(f \circ \tau^{-1}\right)\left(x_{2}\right)$ it follows that $\tau^{-1}\left(x_{1}\right) \sim \tau^{-1}\left(x_{2}\right)$ whenever $f_{0}\left(\tau^{-1}\left(x_{1}\right)\right)=f_{0}\left(\tau^{-1}\left(x_{2}\right)\right) \neq 0$. This proves (2) of the proposition.

Let $\mathbf{Z}_{k}$ be identified with a dense subset of $X$. For $1 \leq i \leq k$ define the cluster sets $X_{i+}$ and $X_{i-}$ by

$$
X_{i+}=\bigcap_{j=1}^{\infty} \overline{\{(n, i): n \geq j\}} \text { and } \quad X_{i-}=\bigcap_{j=1}^{\infty} \overline{\{(n, i): n \leq-j\}}
$$

Proposition 5.3.2. There exists a function $f$ in $\mathscr{A}$ such that $\left.f\right|_{X_{i \pm}}=a_{i \pm}$ if and only if $a_{i \pm}=0$ whenever $X_{i \pm} \cap X_{0} \neq \varnothing$ and $a_{i \pm}=a_{j \pm}$ whenever there exists $x_{1} \in X_{i \pm}, x_{2} \in X_{j \pm}$ such that $x_{1} \sim x_{2}$.

Proof. A function $f$ in $\mathscr{A}$ such that $\left.f\right|_{X_{i \pm}}=a_{i \pm}$ clearly satisfies the requirement.

Conversely, let $\left\{a_{i \pm}\right\}_{1}^{k}$ satisfy the given conditions. Let $F=\bigcup_{i=1}^{k} X_{i \pm}$ and $\psi: X \rightarrow Y$ be the quotient map. Because $X$ is compact, $F$ is closed in $X$ and $\psi$ is continuous it follows that $\psi(F)$ is closed in the Hausdorff space $Y$. If $\tilde{f}$ is such that $\left.\tilde{f}\right|_{\psi\left(X_{i \pm}\right)}=a_{i \pm}$ then $\tilde{f}$ is continuous on $\psi(F)$. By the Tietze extension theorem there is an extension $\tilde{\tilde{f}}$ defined on $Y$ such that $\tilde{\tilde{f}}\left(p_{0}\right)=0$. Hence $f$ exists in $\mathscr{A}$ satisfying the required conditions.

Lemma 5.3.3. If $X_{i \pm} \cap\left\{x \in X: f_{0}(x)=0\right\} \neq \varnothing$ for some $i$ such that $1 \leq i \leq k$, then the marked graph $G$ associated with $T$ has a (0) vertex corresponding to the end $T_{i \pm}$.

Proof. Suppose $x$ is in the intersection of the two sets. Then there is a net $\left\{n_{\alpha}\right\}$ in $\mathbf{Z}$ such that $\left(n_{\alpha}, i\right) \rightarrow x$ and $n_{\alpha} \rightarrow \pm \infty$ as $\alpha \rightarrow \infty$. Because $f_{0}\left(n_{\alpha}, i\right) \rightarrow 0$ and $f_{0}\left(n_{\alpha}, i\right)=w_{n_{\alpha}}^{i}$ it follows that zero is a cluster point for the weight sequence of $T_{i \pm}$. Therefore the corresponding vertex in the graph $G$ associated with $T$ is assigned a $(0)$. 
Lemma 5.3.4. If $x_{1}$ in $X_{i \pm}$ and $x_{2}$ in $X_{j \pm}$ are such that $x_{1} \sim x_{2}$, then either the corresponding ends $T_{i \pm}$ and $T_{j \pm}$ of $T$ are vertices incident on an edge of the marked graph $G$ associated with $T$ or both are (0) vertices in $G$.

Proof. If $x_{1} \sim x_{2}$, then Proposition 5.3.1 will be applied. Suppose that $n_{1}$ is the smallest nonnegative integer such that $f_{0}\left(\tau^{n_{1}}\left(x_{1}\right)\right)=0$. Then $\tau^{m}\left(x_{1}\right) \sim$ $\tau^{m}\left(x_{2}\right)$ for $m=0,1,2, \ldots, n_{1}$ and $f_{0}\left(\tau^{n_{1}}\left(x_{2}\right)\right)=0$. Note that $X_{i \pm}$ is invariant under $\tau$ and $\tau^{-1}$. Hence from Lemma 5.3.3 the two ends correspond to (0) vertices in $G$. A similar argument can be used to get the same conclusion when $n_{1}$ is the smallest nonnegative integer such that $f_{0}\left(\tau^{-n_{1}}\left(x_{1}\right)\right)=0$.

Suppose $f_{0}\left(\tau^{n}\left(x_{1}\right)\right) \neq 0$ or $f_{0}\left(\tau^{n}\left(x_{2}\right)\right) \neq 0$ for $n=0,1,2, \ldots$ then $\tau^{n}\left(x_{1}\right) \sim \tau^{n}\left(x_{2}\right)$ for $n=0,1,2, \ldots$. Let $\left\{n_{\alpha}\right\}$ and $\left\{m_{\beta}\right\}$ be nets such that $n_{\alpha} \rightarrow \pm \infty, m_{\beta} \rightarrow \pm \infty,\left(n_{\alpha}, i\right) \rightarrow x_{1}$ and $\left(m_{\beta}, j\right) \rightarrow x_{2}$. Now $\left(n_{\alpha}+s, i\right) \rightarrow$ $\tau^{s}\left(x_{1}\right)$ and $\left(m_{\beta}+s, j\right) \rightarrow \tau^{s}\left(x_{2}\right)$ follow from the relation $\tau(n, i)=(n+1, i)$. Because $\tau^{s}\left(x_{1}\right) \sim \tau^{s}\left(x_{2}\right)$ for each $s, f_{0}\left(\tau^{s}\left(x_{1}\right)\right)=f_{0}\left(\tau^{s}\left(x_{2}\right)\right)$ for each $s$. Therefore $f_{0}\left(n_{\alpha}+s, i\right)-f_{0}\left(m_{\beta}+s, j\right) \rightarrow 0$ as $n_{\alpha}$ and $m_{\beta}$ approach $\pm \infty$. Hence, given $\varepsilon>0$ and a positive integer $s$ there exist integers $n_{\alpha}$ and $m_{\beta}$ such that $\left|w_{n_{\alpha}+l}^{i}-w_{m_{\beta}+l}^{j}\right|<\varepsilon$ for $l=1,2, \ldots, s$ where $f_{0}\left(n_{\alpha}+l, i\right)=w_{n_{\alpha}+l}^{i}$ and $f_{0}\left(m_{\beta}+l, j\right)=w_{m_{\beta}+l}^{j}$. This proves that the ends $T_{i \pm}$ and $T_{j \pm}$ are block balanced and form end vertices of an edge in $G$. Suppose $f_{0}\left(\tau^{-n}\left(x_{1}\right)\right) \neq 0$ or $f_{0}\left(\tau^{-n}\left(x_{2}\right)\right) \neq 0$ for $n=0,1,2, \ldots$ then $\tau^{-n}\left(x_{1}\right) \sim \tau^{-n}\left(x_{2}\right)$ for $n=$ $0,1,2, \ldots$. A similar argument shows that the corresponding ends of $T$ are block balanced.

If $\mathscr{I}$ is the ideal in $\mathscr{A}$ generated by $f_{0}$, then $\mathscr{I}=\overline{\left\{f_{0} f: f \in \mathscr{A}\right\}}$ and the closed subset of $Y$ associated with $\mathscr{I}$ is $\left\{y \in Y: f_{0}(y)=0\right\}$.

Lemma 5.3.5. If $f$ is in $\mathscr{I}$, then $\mathscr{W} f$ belongs to $C^{*}(T)$.

Proof. Suppose that $\mathscr{D}_{1}$ and $\mathscr{D}_{2}$ in $\mathscr{A}$ correspond to $f_{1}$ and $f_{2}$ respectively. Then $T \mathscr{D}_{1}, \mathscr{D}_{1} T$ and $\mathscr{D}_{1} T \mathscr{D}_{2}$ belong to $C^{*}(T)$ and correspond to $\mathscr{W} f_{0} f_{1}$, $\mathscr{W}\left(f_{1} \circ \tau\right) f_{0}$ and $\mathscr{W}\left(f_{1} \circ \tau\right) f_{0} f_{2}$. Therefore for every $f$ in $\mathscr{I}$, $\mathscr{W} f$ must belong to $C^{*}(T)$.

5.4. Conclusion of the proof. The results from 5.3 allow us to finish the proof of the necessity. Suppose $C$ is a component of $G$ with $p(+)$ vertices and $q(-)$ vertices and no (0) vertex where $p \neq q$. Let $f$ be a function that vanishes on the cluster sets of the ends with vertices not in $C$. The function $f$ can be extended to a continuous function taking the value 1 on the cluster sets of the ends with vertices in $C$. By Proposition 5.3.2 there is such an extension in $\mathscr{A}$. Moreover this extension, also denoted by $f$, belongs to $\mathscr{I}$. From Lemma 5.3.5, $\mathscr{W} f$ is in $C^{*}(T)$. This $\mathscr{W} f$ is a compact perturbation of

$$
\underbrace{U \oplus U \oplus \cdots \oplus U}_{p \text { times }} \oplus \underbrace{U^{*} \oplus U^{*} \oplus \cdots \oplus U^{*}}_{q \text { times }} \oplus 0
$$

where 0 corresponds to the vertices not in $C$. Because

$$
\underbrace{U \oplus U \oplus \cdots \oplus U}_{p \text { times }} \oplus \underbrace{U^{*} \oplus U^{*} \oplus \cdots \oplus U^{*}}_{q \text { times }}
$$

is Fredholm with nonzero index, $T$ is not quasidiagonal. 
Remark. In [8] D. Hadwin generalized part of Smucker's theorem to the context of $C^{*}$-algebraic crossed products of $C(X)$ by $\mathbf{Z}$. His result specifies which irreducible representations associated to orbits are quasidiagonal. If $T$ is a weighted shift, $C^{*}(T)$ is such a crossed product when $T$ is bilateral and invertible; but in general it is only a "generalized crossed product" in the sense of some not yet published work of L. G. Brown [6]. [This replaces an automorphism of a $C^{*}$-algebra $\mathscr{B}$ by a strong Morita equivalence of one ideal of $\mathscr{B}$ with another ideal of $\mathscr{B}$. For the case of weighted shifts the Morita equivalence arises from an actual isomorphism between the ideals generated by $f_{0}$ and $f_{0} \circ \tau^{-1}$.]

Our exposition in $\S 5$ can perhaps serve as an introduction to the theory of generalized crossed products (we are told that the example of weighted shifts motivated this theory) as well as an indication of how Hadwin's result could be generalized to this context. Of course, our result relates to the quasidiagonality of a finite direct sum of irreducible representations rather than a single irreducible representation. Smucker proved the necessity of his condition without the need for detailed analysis of $C^{*}(T), \mathscr{A}$ or $\mathscr{I}$ and we have chosen to give more such analysis than necessary for the reasons indicated.

\section{ACKNOWLEDGMENTS}

The author would like to thank Professor Lawrence G. Brown for his guidance during the preparation of this work, Professor Lowell W. Beineke for providing the references on marked graphs, and the referee for some helpful comments.

\section{REFERENCES}

1. C. Apostol, C. Foias, and D. Voiculescu, Some results on non-quasitriangular operators, IV, Rev. Roumaine Math. Pures Appl. 18 (1973), 487-514.

2. L. W. Beineke and F. Harary, Consistency in marked digraphs, J. Math. Psych. 18 (1978), 260-269.

3. _ Consistent graphs with signed points, Riv. Mat. Sci. Econom. Social. 1 (1978), 81-88.

4. J. A. Bondy and U. S. R. Murty, Graph theory with applications, Macmillan, London and Elsevier, New York, 1976.

5. L. G. Brown, The universal coefficient theorem for Ext and quasidiagonality, Operator Algebras and Group Representations, Vol. 1, Pitman Monographs and Studies in Math. 17, Pitman Adv. Publ. Program, Boston, Mass., 1984, pp. 60-64.

6. _ Generalized crossed products of $C^{*}$-algebras (in preparation).

7. J. B. Conway, A course in functional analysis, Graduate Texts in Math. 96, Springer-Verlag, New York, 1985.

8. D. Hadwin, Strongly quasidiagonal $C^{*}$-algebras, J. Operator Theory 18 (1987), 3-18.

9. P. R. Halmos, Quasitriangular operators, Acta Sci. Math. (Szeged) 29 (1968), 283-293.

10. __ Ten problems in Hilbert space, Bull. Amer. Math. Soc. 76 (1970), 887-933.

11. _ _ A Hilbert space problem book, 2nd ed., Graduate Texts in Math. 19, Springer-Verlag, New York, 1982.

12. D. A. Herrero, Approximation of Hilbert space operators, Vol. 1, Research Notes in Math. 72, Pitman Adv. Publ. Program, Boston, Mass., 1982.

13. G. Luecke, A note on quasidiagonal and quasitriangular operators, Pacific J. Math. 56 (1975), 179-185.

14. C. M. Pearcy, Some recent developments in operator theory, CBMS Regional Conf. Series Math., no. 36, Amer. Math. Soc., Providence, R.I., 1978. 
15. A. L. Shields, Weighted shift operators and analytic function theory, Math. Surveys, no. 13, edited by C. M. Pearcy, Amer. Math. Soc., Providence, R.I., 1974.

16. R. A. Smucker, Quasidiagonal and quasitriangular operators, Dissertation, Indiana Univ., 1973.

17. __, Quasidiagonal weighted shifts, Pacific J. Math. 98 (1982), 173-182.

Department of Mathematics, Purdue University, West Lafayette, Indiana 47907

Current address: Department of Mathematics, Central Michigan University, Mount Pleasant, Michigan 48859

E-mail address: 343qhrp@cmuvm.bitnet 